\title{
Female athletes and menstrual disorders: a pilot study
}

\author{
Laura Stefani ${ }^{1}$ \\ Giorgio Galanti ${ }^{1}$ \\ Silvia Lorini ${ }^{1}$ \\ Giada Beni ${ }^{2}$ \\ Metella $\mathrm{Dei}^{2}$ \\ Nicola Maffulli ${ }^{3}$
}

1 Sports Medicine Center, Clinical and Experimental Department, Florence, Italy

2 Child and Woman Health Department,

University of Florence, Italy

3 Department of Musculoskeletal Disorders

Faculty of Medicine, Surgery and Dentistry,

University of Salerno, Salerno, Italy;

Centre for Sports and Exercise Medicine

Barts and London School of Medicine and Dentistry, Queen Mary University of London, London, UK

Corresponding author:

Laura Stefani

Sports Medicine Center, Clinical and Experimental

Department

Via delle Oblate, 5

50141 Florence, Italy

E-mail: laura.stefani@unifi.it

\section{Summary}

Background: There is a greater incidence of menstrual disorders in female athletes than in their sedentary counterparts. The menstrual disorder is reported in female athletes suffering from athletic triad syndrome, while few data in those free of this syndrome are available. The study aims to ascertain the presence of menstrual disorders and the eventual relationship with myocardial performance in female athletes practicing different sports.

Methods: A sample of 64 subjects aged $18.5 \pm 2$ was selected and divided into 3 groups ( 37 subjects practicing rhythmic gymnastics, 11 swimmers, and 16 volleyball players). All underwent echocardiography, biompendance analysis, and answered a questionnaire.

Results: All anthropometrics parameters were normal. Few athletes reported menstrual disorders. No association between the presence of menstrual disorders and BMI. All echo results were within the normal range. Cardiac Mass Index (CMI) was nor- mal for all athletes despite in swimmers significantly higher values $\left(90.64 \pm 14.9 \mathrm{~g} / \mathrm{m}^{2}\right)$ compared to the volleyball players $\left(78.25 \pm 14.0 \mathrm{~g} / \mathrm{m}^{2} ; \mathrm{p}<.04\right)$ and rhythmic gymnasts $\left(77.89 \pm 13.4 \mathrm{~g} / \mathrm{m}^{2} ; \mathrm{p}<.009\right)$ were found.

Conclusions: Despite menstrual disorders are represented among female athletes, the eventual relationship with the sport practiced is not so evident. Questionnaire should be used to identify menstrual disorders in non-elite athletically active females.

KEY WORDS: body composition, female athletes, menstrual disorder.

\section{Introduction}

Menstrual disorders are more frequent in women practicing intense physical activity than the general population ${ }^{1,2}$

Menstrual changes are part of the female athletic triad syndrome, consisting on insufficient energy availability, menstrual disorders and decreased bone mineral density3,4. Menstrual disorders are mainly studied within the framework of the female athlete triad ${ }^{3,4}$, and are ascribed to insufficient energy availability in the presence of strenuous physical activity exceeding food energy intake.

The prevalence of the triad varies depending on the type of sport; it is more frequently associated with sports requiring low body mass. This is exacerbated in gymnastics and dance disciplines, which also require the athletes to have an idealized body configuration ${ }^{5-7}$. Few data are available about the prevalence of oligoamenorrhea in female athletes without symptoms related to the triad syndrome, and especially in athletes from sports not typically considered at risk to develop the syndrome.

The purpose of this pilot study is to ascertain whether it is possible to collect data in such athletes. Also, the investigation aimed to verify the possible association of menstrual disorders with body composition and different cardiac functional pattern in athletes practicing different sports.

\section{Materials}

Three groups of female athletes were investigated. All gave their formal consent to participate in the study. The study was conducted ethically according the international standard guidelines ${ }^{8}$. 
Group 1 was composed of 37 rhythmic gymnasts; Group 2 included 11 swimmer, of similar age, and Group 3 included 16 volleyball players. Rhythmic gymnastics has a high static and a low dynamic component, swimming has a medium/low static component and medium/high dynamic component, and volleyball has a high dynamic component ${ }^{9}$.

All female athletes had trained on a regular basis for at least three years before entering the study. All trained at least three hours/day for at least three times a week, and competed at least 3 times per month.

They all underwent clinical and physical examination to exclude disorders of thyroid function, primary ovarian failure and hyperandrogenism. All were asked about use of contraceptive drugs. Subjects with metabolic diseases, such as diabetes or hypertension, were excluded.

\section{Methods}

\section{Body composition measurements}

Height, weight, BMI $\left(\mathrm{kg} / \mathrm{m}^{2}\right)$ (Body Mass Index) and body water composition (by bioimpedance analysis) including Fat Free Mass (FFM), Fat Mass (FM), total body water (TBW), intracellular water (ICW), extracellular water (ECW), PA (phase angle) were measured.

The participants were dressed in minimal clothing during the measurements. All evaluations were performed in the morning, following an overnight fast, with the subjects lying supine on a standard examination couch.

\section{Menstrual status}

To investigate menstrual disorders, a dedicated questionnaire was used.

The first part of the questionnaire concerned the age at menarche, length of the menstrual cycles, and history of amenorrhea.

Primary amenorrhea was diagnosed where there was no onset of menses by age 15 .

Secondary amenorrhea was diagnosed when there was no menstruation for 6 months, or for more than three times the previous cycle length.

Menstrual periods that occurred more than 35 days apart were described as oligomenorrhea. Polymenorrhea was defined in case of menses with a frequency of less than 21 days.

The second part of the questionnaire referred to sport activities: age at the beginning of training, training period, number of training session per week, hours of training per day and per week.

\section{Echocardiographic exam}

A standard 2D echocardiographic exam was also performed at rest by two experts cardiologists sonographers, using the echocardiograph My Lab 50 (Esaote, Italy) equipped with a $2.5 \mathrm{MHz}$ transthoracic echocardiographic probe. According to the ACC/AHA guide- lines ${ }^{10}$, the echocardiographic measurements of the left ventricle (LV) chamber were obtained from the parasternal long-axis view.

They included LV end-systolic and end-diastolic diameters (LVEDd, LVESd), interventricular septum (IVS), posterior wall thickness (PW), and the ejection fraction (EF\%), calculated according to the formula (LVEDdLVESd/LVEDd), for which volumes are substituted by diameters.

By Doppler analysis, the diastolic variables were measured in the presence of a stable RR interval and in three different but sequential measurements from the four chamber view.

They consisted in transmitral flow of $E$ wave and $A$ wave peak velocities, isovolumetric relaxation and deceleration times, and E/A ratio.

The degree of severity of valve insufficiency, described as the extent of the regurgitant jet on a 0 to $4+$ scale, was assessed using the colour-flow mapping method from the four-chamber view, according to the ACC/AHA guidelines ${ }^{10}$.

The left ventricular mass index (LVMI) and left ventricular hypertrophy (LVH) were calculated according to the equation by Devereux ${ }^{11}$.

In addition, the myocardial wall thickness (W/T) ratios index of myocardial remodeling was measured.

\section{Statistical analysis}

All data expressed the mean and standard deviation and are compared using the Student's t test for unpaired data (ANOVA Test). The correlation coefficient ( $r$ ) among the presence of menstrual disorders and the antropometric parameter was also evaluated.

\section{Results}

\section{Body composition and hydration parameters}

All the data from the bioelectrical impedance were obtained using BIA 101 Sport Edition, Akern, Florence, Italy.

These data were calculated in \% and in respect to the weight.

The results were within the normal range, with the exclusion of the total body water, which were below normal range.

Swimmers had significantly higher FFM compared to rhythmic gymnasts and volleyball players. No significant differences in the other parameters related to body hydration were observed among the groups. There was no difference in BMI among the three groups.

\section{Questionnaire data}

The average age of menarche in the rhythmic gymnasts was 12.8 year.

Regarding the length of the menstrual cycle, no girl reported a cycle shorter than 25 days; 9 (24\%) reported 
between 25 and 36 days; and 3 (8.7\%) reported more than 36 days.

One of 37 of these athletes (1\%) reported amenorrhea, while $3(8 \%)$ reported oligomenorrhea.

Only 1 rhythmic gymnast (1\%) reported that a menstrual period had been missing for more than 3 months. One athlete followed a hypocaloric normoproteic diet. Seven rhythmic gymnasts $(8.3 \%)$ reported that they tried to limit their fatty foods intake.

The average age of menarche in the 11 swimmers was 12.7 years.

Regarding the length of the menstrual cycle, no girl reported a cycle shorter than 25 days; 9 (81\%) reported between 25 and 36 days, and 2 (18\%) reported longer than 36 days.

Five swimmers (45.4\%) reported eumenorrhea, and 4 (36\%) oligomenorrhea.

None of them reported any specific restriction in their diet.

The average age at menarche of the 16 volleyball players was 11.9 .

Two $(12.5 \%)$ volleyball players reported a menstrual cycle usually longer than 36 days, compatible with oligomenorrhea.

None reported cycles shorter than 25 days, and none reported amenorrhea.

No volleyball player reported any specific restriction in their diet.

In all groups, none of the girls with menstrual problems has ever consulted a specialist.

One rhythmic gymnast was under estrogen-progestin therapy for skin aliments.

None of the athletes had otherwise been on estrogenprogestin therapy.

A total of 17 (63\%) athletes, 15 of whom were rhythmic gymnasts, reported to be aware of the presence of menses, but this did not exert any influence on their athletic performance.

Regarding the athletes' nutritional habits, only 1 said to follow a specific diet in order to maintain a low weight and an adequate FFM, while 6 said they have limited the consumptions of fatty foods (Tables I, II).

\section{Discussion}

The principal statement of the present pilot study is the female athletes practicing different kind of sports had menstrual disorders, but they were equally distributed among them.

All the female athletes investigated had a relatively low though still normal BMI.

On the contrary, hydration status was below the reference values while the myocardial performance did not was affected and it normal for all.

Despite the presence of menstrual disorders found, none of the girls with menstrual dysfunction had ever consulted a gynecologists.

This support the hypothesis of a functional and not organic origin of the menstrual dysfunction.

A first hypothesis can be that menstrual disorders found can be partially attributed to a chronic and inadequate fluid replacement, especially after recurrence of sweating.

At the same time it suggests that the problem exists, but it is usually underestimated, and an appropriate educational program should be implement to make sure that the reproductive function of these young female athletes is not compromised by their continuing sport activity.

With respect of the current literature, where only female athletes suffering from athletic triad syndrome, the progressive reduction of caloric intake results in worsening of athletic performance have been well investigated, the present study highlights on the contrary the importance to evaluate a different population of female athletes.

Literature reports as body composition can influence athletic performance ${ }^{11-13}$.

Considering that the total boy water amount and tissue distribution could be interpreted as the principal aspect of the nutritional status in athletes, this data give importance of this aspect.

The sample is however small and therefore the eventual conclusions cannot be largely extended: this is the principal limit of the study.

The present investigation needs to be in fact interpreted as a pilot study.

On the contrary, the possible presence of menstrual disorders and their influence on sports performance in case of female athletes not affected by specific athletic triad syndrome, especially if practicing different sports is poorly investigated.

Particularly, no data are available about any eventual relationship with cardiovascular parameters.

According to the literature $2,12,13$ the results show as the

Table I. Anthropometric values.

\begin{tabular}{lllll}
\hline & Group 1 (Rhythmic gymnasts 37) & Group 2 (Swimmers 11) & Group 3 (Volleyball players 16) & $\boldsymbol{P}$ \\
\hline FFM & $75.8 \pm 4.4 \%$ & $77.2^{*} \pm 1.7 \%$ & $72.9 \pm 5 \%$ & $\mathbf{0 . 0 2}$ \\
FM & $25.1 \pm 4.4 \% ;$ & $22.8 \pm 1.6 \%$ & $27 \pm 5 \%$ & $\boldsymbol{N S}$ \\
TBW & $54.8 \pm 3.4 \%$ & $55.8 \pm .2 \%$ & $53.5 \pm 3.8 \%$ & $\boldsymbol{N S}$ \\
BMI & $19.4 \pm 2.4$ & $20.1 \pm 2.1$ & $20.54 \pm 1.9$ & NS \\
\hline
\end{tabular}

Legend: FFM: Fat Free mass; FM:Fat Mass; BMI: Body Mass Index.

${ }^{*} \mathrm{p}<0.05$ for $\mathrm{G} 2$ vs $\mathrm{G} 3$. 
Table II. Echocardiographic characteristics.

\begin{tabular}{llll}
\hline Ecocardiographic characteristics & & \\
\hline & $\begin{array}{l}\text { Group 1 Rhythmic gymnasts } \\
\text { (37 athletes) }\end{array}$ & $\begin{array}{l}\text { Group 2 Swimmers } \\
(11 \text { athletes })\end{array}$ & $\begin{array}{l}\text { Group 3 Volleyball players } \\
\text { (16 athletes) }\end{array}$ \\
\hline EF \% & $62.2 \pm 19.27$ & $68.5 \pm 3.3$ & $67.6 \pm 3.0$ \\
CMI gr/m & $77.8 \pm 13.4^{*}$ & $90.6 \pm 14.9^{* *}$ & $78.25 \pm 14.0^{*}$ \\
IVS mm & $7.8 \pm 0.8$ & $8.3 \pm 1.3$ & $7.9 \pm 0.5$ \\
PW mm & $7.5 \pm 0.8$ & $8.1 \pm 0.8$ & $7.9 \pm 0.6$ \\
LVEDd mm & $44.3 \pm 2.9^{*}$ & $45.7 \pm 3.3^{*}$ & $44.4 \pm 2.7^{*}$ \\
LVESd mm & $26.6 \pm 3.8^{*}$ & $29.1 \pm 1.3^{*}$ & $28.1 \pm 2.5$ \\
E wave (msec) & $89.3 \pm 31.9$ & $99.5 \pm 12.1$ & $100.6 \pm 14.1$ \\
A wave (msec) & $49.3 \pm 19.4$ & $47.6 \pm 15.2$ & $51.6 \pm 15.3$ \\
IVR (ms) & $66.1 \pm 22.4$ & $73.9 \pm 10.8$ & $69.3 \pm 7.4$ \\
DTc(ms) & $158.9 \pm 55.0$ & $167.8 \pm 18.6$ & $166.2 \pm 18.1$ \\
W/T & $0.3 \pm 0.0$ & $0.3 \pm 0.0$ & $0.3 \pm 0.0$ \\
RV & $20 \pm 2.3$ & $21 \pm 2.2$ & $22.4 \pm 2.2$ \\
\hline
\end{tabular}

Legend: EF: ejection fraction; CMI: cardiac mass index; IVS: interventricular septum; PW: posterior wall; LVEDd: left ventricle diastolic diameter; LVESd: left ventricle systolic diameter; DTc: deceleration time; IVRT: isovolumic relaxation time; W/T: Wall Thickness ratio; RV: right ventricle. Statistical significance: ** $p<.001 \mathrm{G} 2>\mathrm{G} 1>\mathrm{G} 3 ;{ }^{*} \mathrm{p}<.05 \mathrm{G} 3>\mathrm{G} 1>\mathrm{G}$.

menstrual disorders are not associated to an evidence of impaired cardiac function.

The echocardiographic parameters were, as expected, different according to the different kind of sport practiced, especially considering the cardiac mass index (BMI) in the swimmers, who undertook mainly prolonged aerobic activities, compared to the other athletes.

In all athletes, the echocardiographic variables were within normality, and they were not related to lean mass, total body water, or the presence of menstrual disorders.

The possible impact of the psychological stresses of athletic training and competition in young female athletes, is a very important aspect in this field, however it has not been considered as aim in this investigation, but we acknowledge that stress may be associated with menstrual disorders.

This is potentially an additional field of interest. Future perspectives should include more evidence of this potential relationship.

Equally, the effect of possible musculoskeletal injuries on menstrual disorders needs to be more evaluated in greater depth.

This component has not been absolutely considered as a part of the present investigation as well as the eventual role of stress competition in young female athletes.

\section{Conclusion}

Nutrition habits should be investigated in female athletes even though they do not present the evident stigmata of the athletic triad syndrome ${ }^{14,15}$. Authors report as menstrual disorders are common among female athletes ${ }^{16}$, and therefore the present study seems to be partially in disagreement with the data of the current literature.

Our data highlight the possible influence of poor hydration $^{17}$. In these athletes, there is no other evidence, with the exclusion of a possible inadequate caloric intake. A larger cohort of subjects needs to be however investigated in order to sustain this hypothesis.

In general it seems to be important the investigation of the lifestyle and the eating habits of female athletes using an appropriate dietary questionnaires.

These methods, largely used in sports medicine in many different clinical conditions, where the examination of the amount of physical activity and of the daily the lifestyle is very important ${ }^{18,19}$ in adolescent, could be helpful as data's collection to identify the eventual menstrual disorders in elite and non-elite athletically active females and therefore to permit a possible intervention in the potential incorrect lifestyle habits.

\section{Conflict of interests}

The Authors declare that they have no conflict of interests regarding the publication of this paper.

\section{References}

1. American Academy of Pediatrics (Committee on Sports Medicine and Fitness), Medical concerns in the female athlete. Pediatrics. 2000;(106):610-613. 
2. Dadgostar H, Razi M, Aleyasin A, Alenabi T, Dahaghin S. The relation between athletic sports and prevalence of amenorrhea and oligomenorrhea in Iranian female athletes. Sports Med Arthrosc Rehabil Ther Technol. 2009;1-7.

3. Nattiv A, Loucks AB, Manore MM, Sanborn CF, Sundgot-Borgen J, Warren MP. (American College of Sports Medicine) The female athlete triad. MSSE. 2007;1867-1882.

4. Christo K, Prabhakaran R, Lamparello B, et al. Bone metabolism in adolescent athletes with amenorrhea, athletes with eumenorrhea, and control subjects. Pediatrics. 2008;1127-1136.

5. American Academy of Pediatrics (Committee on Sports Medicine and Fitness), Promotion of healthy weight-control practices in young athletes, Pediatrics. 2005;116:1557-1564.

6. Beals KA, Manore MM. Subclinical eating disorders in physically active women. Topics in Clinical Nutrition. 1999;14:14-29.

7. Frisch RE, Gotz-Welbergen AV, Mc Arthur JW, et al. Delayed menarche and amenorrhea of college athletes in relation to age of onset of training. JAMA. 1981;246:1559-1563.

8. Padulo J, Oliva F, Frizziero A, Maffulli N. Muscles, Ligaments and Tendons Journal. Basic principles and recommendations in clinical and field science research:2016 update. MLTJ. 2016;6 (1):1-5.

9. Mitchell JH, Haskell W, Snell P, Van Camp ST. Task force 8: Classification of sports. JACC. 2005;1364-1367.

10. Douglas PS, Garcia MJ, Haines DE, et al. ACCF/ASE/ AHA/ASNC/HFSA/HRS/SCAI/SCCM/SCCT/SCMR 2011 Appropriate use criteria for echocardiography. JASE. 2011; 24:229-267.
11. Devereux RB. Detection of left ventricular hypertrophy by Mmode echocardiography. Anatomic validation, standardization, and comparison to other methods. Hypertension. 1987; 9:2:II9-26.

12. De Souza MJ, Williams NI. Physiological aspects and clinical sequelae of energy deficiency and hypoestrogenism in exercising women. Hum Reprod Update. 2004;5:433-448.

13. Dummer GM, Rosen LW, Heusner WW, Roberts PJ, Counsilman JE. Pathogenic weight-control behaviors of young competitive swimmers. The Physician and Sports medicine. 1987;5:22-27.

14. Loucks $A B$. The female athlete triad: Do female athletes need to take special care to avoid low energy availability? MSSE. 2006;1694-1700.

15. Torstveit Klungland M, Sundgot-Borgen J. The female athlete triad exists in both elite athletes and controls. MSSE. 2005 1449-1459.

16. Manore MM. Dietary recommendations and athletic menstrual dysfunction. Sports Med. 2002;32:887-901.

17. Maïmoun L, Georgopoulos NA, Sultan C. Endocrine Disorders in Adolescent and Young Female Athletes: Impact on Growth, Menstrual Cycles, and Bone Mass Acquisition. J Clin Endocrinol Metab. 2014;99(11):4037-4050.

18. Loucks AB. Exercise training in the normal female. Sports Endocrinology. 2000;165-180.

19. Galanti G, Stefani L, Petri C, Mascherini G. Primary prevention and lifestyles: physical exercise. IEMJ. 2015;10:S 22-30. 